\title{
Linear models for control of cavity flow oscillations
}

\author{
By CLARENCE W. ROWLEY ${ }^{1}$, DAVID R. WILLIAMS ${ }^{2}$, \\ TIM COLONIUS ${ }^{3}$, RICHARD M. MURRAY ${ }^{3}$ AND \\ DOUGLAS G. MACMYNOWSKI \\ ${ }^{1}$ Mechanical and Aerospace Engineering, Princeton University, Princeton, NJ 08544, USA \\ ${ }^{2}$ Mechanical, Materials, and Aerospace Engineering, Illinois Institute of Technology, \\ Chicago, IL 60616, USA \\ ${ }^{3}$ Division of Engineering and Applied Science, California Institute of Technology, \\ Pasadena, CA 91125, USA
}

(Received 21 January 2004 and in revised form 19 July 2005)

Models for understanding and controlling oscillations in the flow past a rectangular cavity are developed. These models may be used to guide control designs, to understand performance limits of feedback, and to interpret experimental results. Traditionally, cavity oscillations are assumed to be self-sustained: no external disturbances are necessary to maintain the oscillations, and amplitudes are limited by nonlinearities. We present experimental data which suggests that in some regimes, the oscillations may not be self-sustained, but lightly damped: oscillations are sustained by external forcing, such as boundary-layer turbulence. In these regimes, linear models suffice to describe the behaviour, and the final amplitude of oscillations depends on the characteristics of the external disturbances. These linear models are particularly appropriate for describing cavities in which feedback has been used for noise suppression, as the oscillations are small and nonlinearities are less likely to be important. It is shown that increasing the gain too much in such feedback control experiments can lead to a peak-splitting phenomenon, which is explained by the linear models. Fundamental performance limits indicate that peak splitting is likely to occur for narrow-bandwidth actuators and controllers.

\section{Introduction}

Recent experiments using feedback to control cavity oscillations have met with limited success. Some early flow-control experiments (Cattafesta et al. 1999; Williams, Fabris \& Morrow 2000) suppressed individual Rossiter tones by up to $20 \mathrm{~dB}$ with active feedback; however, other tones were unaffected or enhanced by the control system. When the control gains were increased to achieve greater suppression, new frequencies would appear in the spectrum, or the suppressed peak would split. These effects were undesirable, and unpredictable. The goal of this paper is to use physicsbased models to understand these effects, guide future control designs, and understand any fundamental performance limits, for a given choice of sensor and actuator.

The usual description of cavity oscillations involves self-sustained oscillations, caused by the familiar Rossiter mechanism (Rossiter 1964; Tam \& Block 1978; Rowley, Colonius \& Basu 2002): small disturbances are amplified by the shear layer, and produce acoustic waves when they impinge on the downstream corner; these 


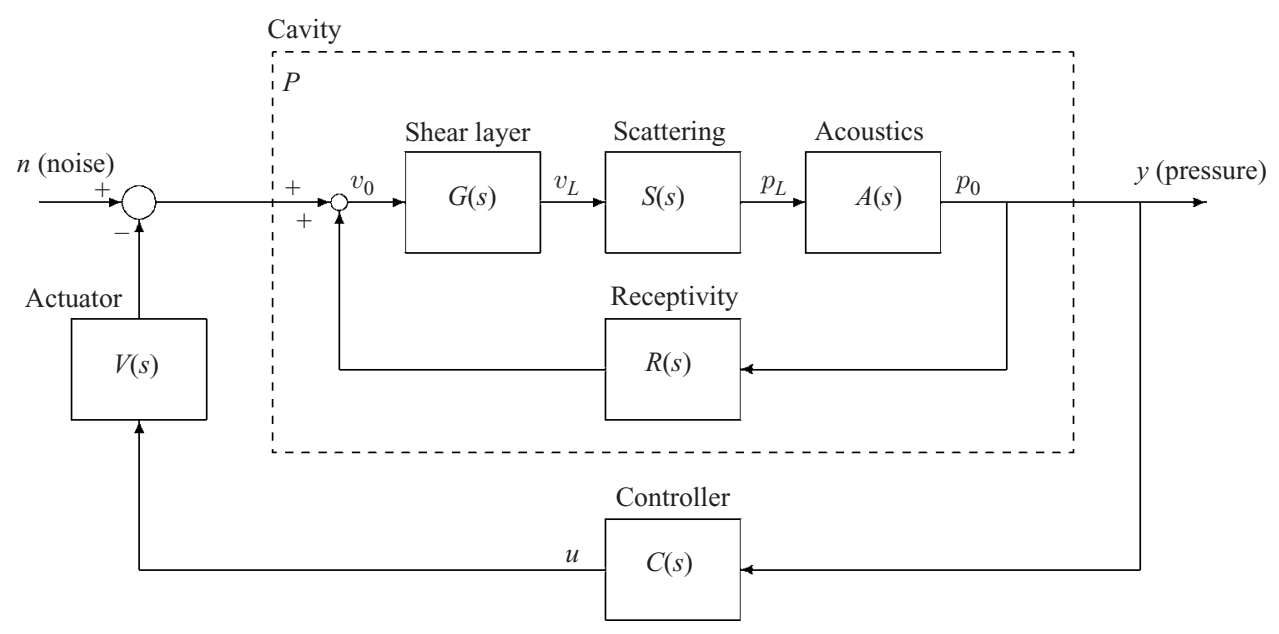

Figure 1. Block diagram of a cavity model.

acoustic waves then propagate upstream and excite further instabilities in the shear layer, leading to self-forcing. In the absence of any external forcing, the cavity would continue to oscillate. The cavity behaves as a dynamical system with a stable limit cycle about an unstable equilibrium point (a steady solution of Navier-Stokes). The amplitude of the oscillations is determined by nonlinearities, such as saturation of instabilities in the shear layer.

By contrast, we demonstrate that for many conditions where oscillations are observed, the cavity behaves as a stable lightly damped system. The flow amplifies noise at certain resonant frequencies, but if the external forcing were removed, the oscillations would disappear. Purely linear models may be used to describe this mechanism, as the final amplitude of oscillations is determined by the amplitude of the forcing disturbances (e.g. boundary-layer turbulence, or wall roughness), and by the linear gain of the system. Nonlinearities, such as saturation of instability waves in the shear layer, may still be present in this mechanism, and will also affect the final amplitude of oscillations, but they are not necessary to explain finite-amplitude oscillations. We show that the previously noted performance limitations of feedback control are explained by this alternative view of cavity oscillations, and note that controllers can be designed to minimize the adverse effects.

The paper is organized as follows: we present the physics-based linear model in $\S 2$, then describe some experimental results in $\S 3$. In $\S 4$, we use the model to explain a peak-splitting phenomenon observed in the experiment, and to understand some fundamental limitations of any feedback controller used to suppress the oscillations.

\section{Analytical model}

The cavity dynamics are modelled after the familiar Rossiter mechanism described in $\S 1$. A block diagram of the model is shown in figure 1 , where we represent each component of the physical mechanism as a separate transfer function. Here, $G(s)$ represents the shear-layer transfer function, i.e. the transfer function from velocity disturbances $v_{0}$ at the leading edge to velocity disturbances $v_{L}$ at the trailing edge. Transfer functions for acoustic scattering, propagation and receptivity are given by $S$, $A$ and $R$, and in the diagram, $p_{0}$ and $p_{L}$ denote pressure disturbances at the leading and trailing edges, respectively. 
The other transfer functions depicted in figure 1 represent the influence of a controller and an actuator (for instance, a zero net mass flux jet or oscillating flap at the cavity leading edge). The controller transfer function, which we choose, is given by $C(s)$, and the actuator dynamics are described by a transfer function $V(s)$. In this diagram, the output $y$ is the pressure at the upstream lip of the cavity, which in an experiment may be measured by a pressure transducer. The plant is excited by external noise (e.g. turbulent boundary-layer fluctuations), modelled by an input disturbance $n$.

The overall transfer function for the cavity is then

$$
P(s)=\frac{A S G}{1-R A S G} .
$$

For the purposes of studying the dynamical features of this model, we ignore the actuator dynamics, setting $V(s)=1$. (These actuator dynamics may, in principle, be measured from the experiment, and once measured, their effects may be inverted out of the control laws we obtain.) Theoretical models for the remaining transfer functions are discussed below.

\subsection{Shear layer}

The shear-layer transfer function $G(s)$ may be determined from linear stability theory. We begin with velocity profiles measured in experiments by Williams et al. (2000), shown in figure 2. These profiles are from an experiment with Mach number $M=0.34$, in a cavity with aspect ratio $L / D=5$, where $L$ and $D$ are the cavity length and depth, respectively, as shown in figure 3 . The upstream boundary layer is turbulent, with a momentum thickness of $3 \mathrm{~mm}$, measured with a Pitot tube and a hot wire. Figure 2 shows the experimental data along with hyperbolic tangent profiles with the same vorticity thickness. Here, $x$ is the streamwise direction, and $y$ is the wall-normal direction, with the origin at the upstream cavity corner. The spreading rate of the shear-layer is determined from a linear fit to the data, and used as an input to a linear stability calculation to determine the amplification and phase of shear-layer disturbances. Because the profiles are slowly varying in the streamwise direction, a locally parallel analysis is used at each streamwise location (for details of the linear stability calculation, see Rowley et al. 2002). We then fit a high-order rational function to the resulting transfer function (with little loss of accuracy), and the result is shown in figure 2. Using this rational function approximation, we may immediately obtain a state-space realization of the transfer function, and apply standard tools from control theory to the resulting model.

As a simpler alternative, we also consider the shear layer modelled as a second-order system with a time delay

$$
G(s)=G_{0}(s) \exp \left(-s \tau_{s}\right)=\frac{\omega_{0}^{2}}{s^{2}+2 \zeta \omega_{0} s+\omega_{0}^{2}} \exp \left(-s \tau_{s}\right),
$$

where $\omega_{0}$ is the natural frequency of the second-order system (e.g. this may be chosen to be the frequency of the most unstable Kelvin-Helmholtz mode), and $\zeta$ is the damping, related to the maximum amplification. The time delay $\tau_{s}$ is the convection time for a disturbance to travel the length of the cavity, and is given by $\tau_{s}=L / c_{p}$, where $c_{p}$ is the mean phase speed.

The purpose of the second-order model (2.2) is not to fit the linear stability results perfectly, but to provide a simple model that captures the same general features: unity gain at low frequencies, amplification at mid frequencies, and attenuation at high 

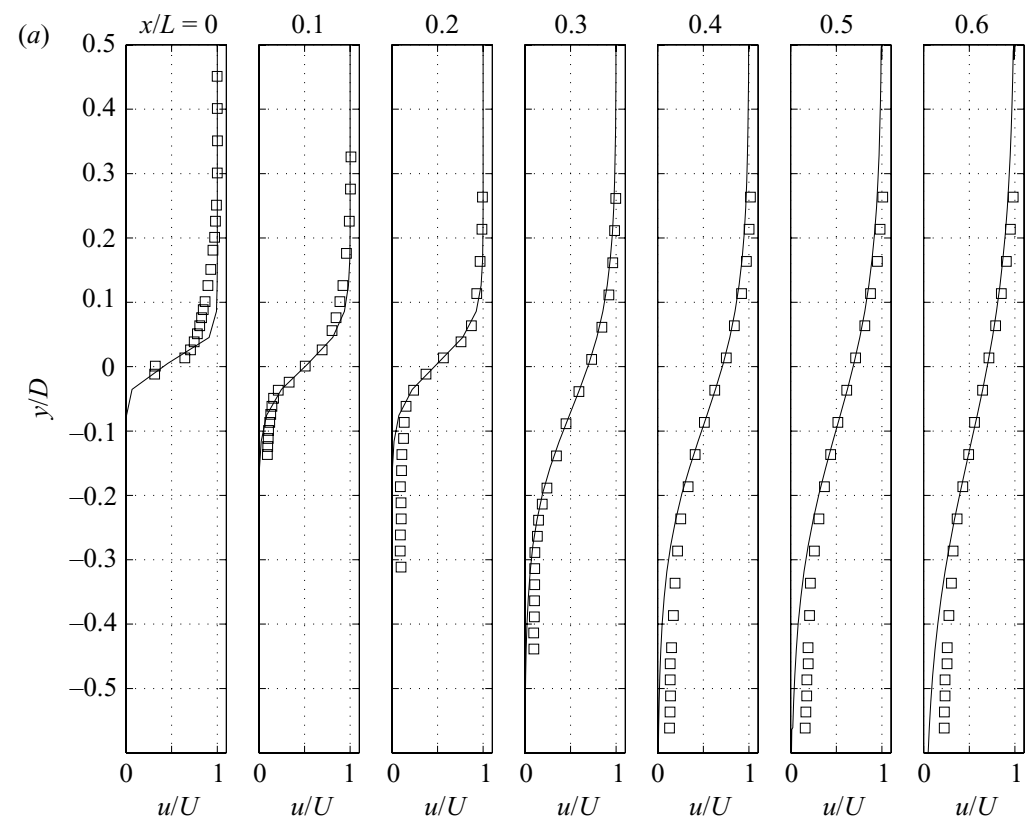

(b)

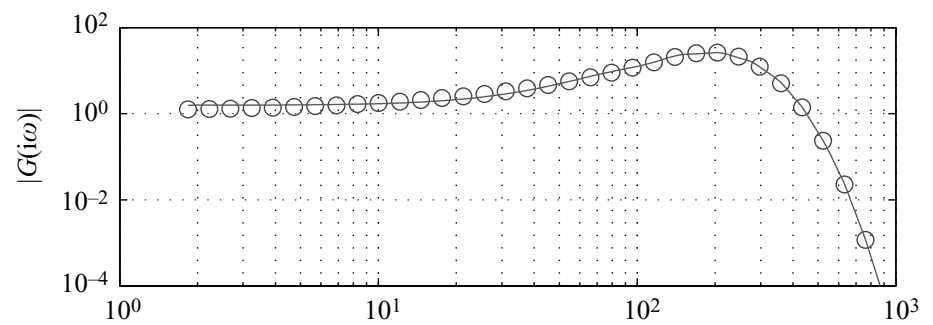

(c)

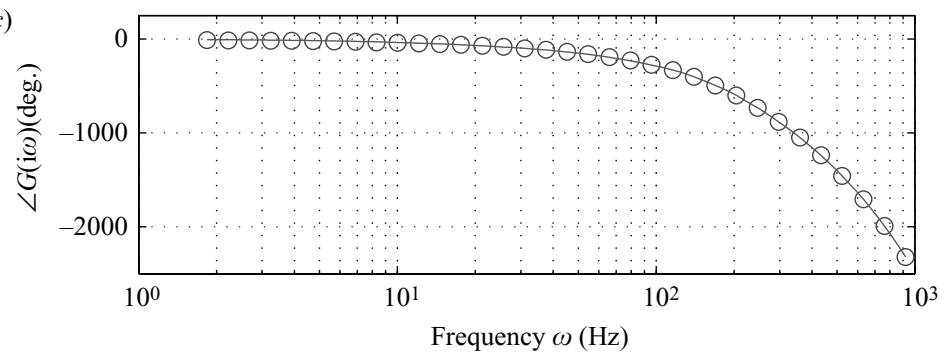

FIGURE 2. (a) Velocity profiles for the cavity shear layer. Hot-wire measurements ( $\square$ ) and tanh profiles with same vorticity thickness and deflection (-). (b) Bode plot of shear-layer transfer function $G(s)$, determined from linear stability theory $(\bigcirc)$, and twentieth-order rational function fit (-).

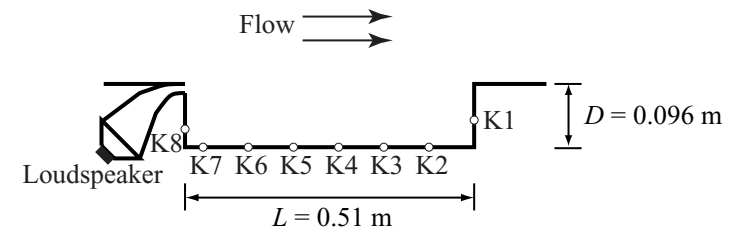

FIGURE 3. Diagram of experimental apparatus (side view). Location of Kulite pressure transducers is indicated by $\mathrm{K} 1-\mathrm{K} 8$. 
frequencies, with an appropriate phase delay. In addition, its adjustable parameters allow the model to be tuned to match specific experimental results, and give insight into the effects of the various parameters. Note that for control, we need the model to be accurate only in the frequency range over which the controller acts, and this model may be tuned to arbitrarily high accuracy in a narrower range of frequencies.

\subsection{Acoustics}

The model we use for acoustic propagation in the cavity consists of a simple timedelay, with reflection:

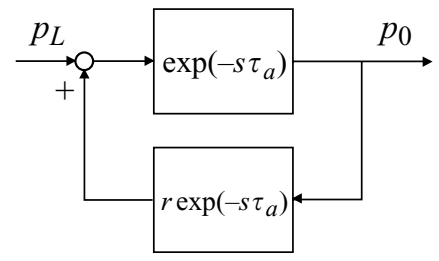

$$
A(s)=\frac{\exp \left(-s \tau_{a}\right)}{1-r \exp \left(-2 s \tau_{a}\right)}
$$

Here, $\tau_{a}=L / a$ is a time delay which represents the acoustic lag between the trailing edge and the leading edge (here, $L$ is the cavity length and $a$ is the sound speed inside the cavity). An acoustic wave emanating from the downstream corner $x=L$ propagates upstream, and some of it reflects off the upstream wall, propagates downstream, and again reflects off the downstream wall. The reflection coefficient $r$ measures the total efficiency of the reflection process, including losses via acoustic radiation to the far field (e.g. if both reflections are perfect, with no radiation to the far field, then $r=1$; if upstream and downstream reflections each reduce the amplitude by 0.5 , then $r=0.25$ ). This model therefore captures longitudinal modes of acoustic resonance, but ignores transverse modes. For $r=0$, the model is a pure time delay, and for $0<r<1$, the Bode magnitude plot of $A(s)$ shows resonant peaks at the fundamental frequency $1 /\left(2 \tau_{a}\right)$ and its harmonics. Note that these acoustic resonances are not the Rossiter frequencies, but they may influence the mode selection, determining which Rossiter mode is dominant, as described in Williams et al. (2000).

\subsection{Scattering and receptivity}

Scattering and receptivity effects at the trailing and leading edge, respectively, are the least simple to model. They have been studied by Crighton (1992) for edge tones, and Kerschen \& Tumin (2003) for cavity flows. In Rossiter's empirical formula for predicting cavity frequencies, the scattering and receptivity effects are treated together as a simple phase lag, independent of frequency. Here, we follow Rossiter's approach and model scattering and receptivity each as complex constants. This approximation is justified since the strongest frequency dependence of the amplitude occurs in the shear layer (Kelvin-Helmholtz instability), and the strongest frequency dependence of the phase results from time delays in the shear layer and acoustics, as discussed above. For a more accurate model, the scattering and receptivity results of Kerschen \& Tumin (2003) could be employed.

\subsection{Overall cavity model}

The overall cavity transfer function $P$ is formed from equation (2.1). To gain some insight into the model, first we consider some special cases. In particular, for certain choices of parameters, we recover the Rossiter formula for the frequencies of oscillation. For the shear-layer model (2.2), suppose $G(s)=\exp \left(-s \tau_{s}\right)$, and take $\tau_{s}=L / c_{p}$, with $c_{p} / U=\kappa$. Assuming $R=1$ and $S=\exp (-\mathrm{i} 2 \pi \gamma)$, a constant phase, 
with no reflections $(r=0)$ in the acoustic model (2.3), the overall transfer function becomes

$$
P(s)=\frac{\exp (-\mathrm{i} 2 \pi \gamma) \exp \left(-s\left(\tau_{s}+\tau_{a}\right)\right)}{1-\exp (-\mathrm{i} 2 \pi \gamma) \exp \left(-s\left(\tau_{s}+\tau_{a}\right)\right)},
$$

which has poles at $s=\mathrm{i} \omega$, with

$$
\frac{\omega L}{2 \pi U}=\frac{n-\gamma}{M+1 / \kappa}, \quad n=1,2, \ldots,
$$

which is the familiar Rossiter formula for the frequencies of oscillation. The other features of the model include the effects of longitudinal acoustic modes in the cavity (with $r>0$ ), as well as amplification effects by the shear layer (with $G_{0}(\mathrm{i} \omega) \neq$ const in (2.2)). Note that both of these features primarily affect the amplitude of the loop gain, and hence the stability of each mode, and have only a minor effect (due to their phase) on the overall resonant frequencies.

The advantage of the present model over Rossiter's original formula is, of course, that the present model is dynamically accurate: it does not merely predict resonant frequencies, but predicts how the output (a pressure measurement) evolves in time, given an arbitrary actuator input or external disturbance. We will use these models to explain some features in the experiment described in the next section.

\section{Experimental dynamics}

Experiments were performed using the $3 \mathrm{ft} \times 3 \mathrm{ft}$ subsonic wind tunnel at the United States Air Force Academy in Colorado Springs. A cavity model $0.51 \mathrm{~m}$ long, $0.096 \mathrm{~m}$ deep and $0.38 \mathrm{~m}$ wide was installed in the floor of the test section, and a diagram of the set-up is shown in figure 3.

Eight Kulite pressure transducers were placed along the cavity walls, one on the upstream wall, one on the downstream wall, and six along the floor, approximately equally spaced. All signals were passed through anti-aliasing filters prior to sampling by a digital data acquisition system at $6 \mathrm{kHz}$. The anti-aliasing filters were fourth-order Butterworth filters with a passband of $0.4 \mathrm{~Hz}$ to $2.2 \mathrm{kHz}$.

The flow was forced using zero-net-mass blowing through a slot in the upstream wall of the cavity, shown in figure 3 . The actuator was a pair of $500 \mathrm{~W} 8$ in diameter loudspeakers in an enclosed chamber. Though the actuator injects zero net mass through the slot, a non-zero net momentum is induced by spanwise vortices generated by periodically blowing through the slot (the 'synthetic jet' effect, Smith \& Glezer 1998).

In order to suppress oscillations, both analogue and digital controllers were implemented. The analogue controller consisted of a bandpass filter and a phase shifter. Digital controllers were implemented using a dSPACE interface board, running on a separate computer from the data acquisition system. For the typical controllers that we used, the maximum sample rate was about $20 \mathrm{kHz}$. Specific details of the controllers used are given in $\S 4.2$.

\subsection{Lightly damped vs. self-sustained oscillations}

As mentioned in $\S 1$, two possible mechanisms may lead to finite-amplitude oscillations. The conventional view, as in Tam \& Block (1978) and Rowley et al. (2002), is that the oscillations are self-sustained: in this mechanism, the linearized system (about a steady solution of Navier-Stokes) is unstable, so tiny perturbations will grow in time, and eventually saturate once nonlinearities become important. An alternative view, 

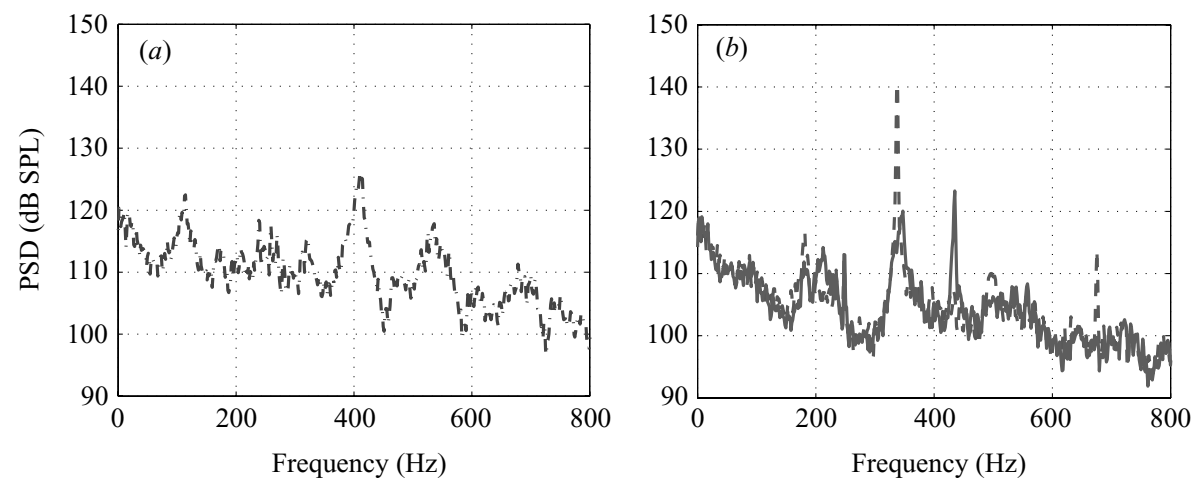

Figure 4. Spectra of the pressure signal from Kulite 7 at Mach numbers $(a) M=0.45(-\cdot-)$, (b) $M=0.34$ unforced (-- $)$, and $M=0.34$ with control $(-)$ (gain =4.0).

considered for combustion instabilities by Mezic \& Banaszuk (2004), is that the system is linearly stable, but lightly damped, and constantly excited by external disturbances. These disturbances are then amplified, causing oscillations at the resonant frequency of the plant, but if the disturbances were removed, the oscillations would also disappear. In practice, disturbances such as boundary-layer turbulence and wall roughness are always present, so this mechanism can also explain persistent oscillations. In this mechanism, nonlinearities may not be important: the amplitude of the oscillations is determined by the amplitude of the excitation noise, and though nonlinearities may still be present, they are not necessary to explain finite-amplitude oscillations. In this section, we demonstrate that the cavity may operate in either the lightly damped or self-sustained regime, depending on the Mach number and other parameters.

Note that in both cases mentioned above, the shear layer is locally convectively unstable (i.e. not absolutely unstable), using the terminology of Huerre \& Monkewitz (1990). However, the flow may still be globally unstable if the acoustic feedback provides a loop gain that is greater than unity. As discussed in Rowley et al. (2002), several parameters affect this loop gain, including the thickness of the upstream boundary layer, and the shear-layer spreading rate, which is influenced by the incident turbulence level (Sarohia 1975). Note that the conditions classified as 'no oscillations' in the numerical investigations in Rowley et al. (2002) may still exhibit oscillations in an experiment, where disturbances are present.

The lightly damped and self-sustained regimes may not be unambiguously identified using only frequency spectra. For instance, spectra from the experiment at two different Mach numbers are shown in figure 4, and it is not clear how we could discern the mechanism from these figures. Both regimes are characterized by peaks at the resonant frequencies, and we cannot tell whether the system is in a (noisy) limit cycle, or whether it is stable, merely amplifying disturbances at certain frequencies. However, it is often possible to distinguish between the two regimes using the probability density function (PDF) of the output signal, bandpass filtered about the frequency of interest (Mezic \& Banaszuk 2004).

If the input disturbances have a Gaussian distribution, the PDF of a stable linear system excited by these disturbances will also be Gaussian. By contrast, the PDF of an observable from a limit cycling system (say $y(t)=\sin (t)$ ) will typically have two peaks, because the system typically spends more time near the extrema of the limit cycle. Note that observables from more complicated periodic orbits might have more complicated PDFs, but generically these also will not be Gaussian, and will 

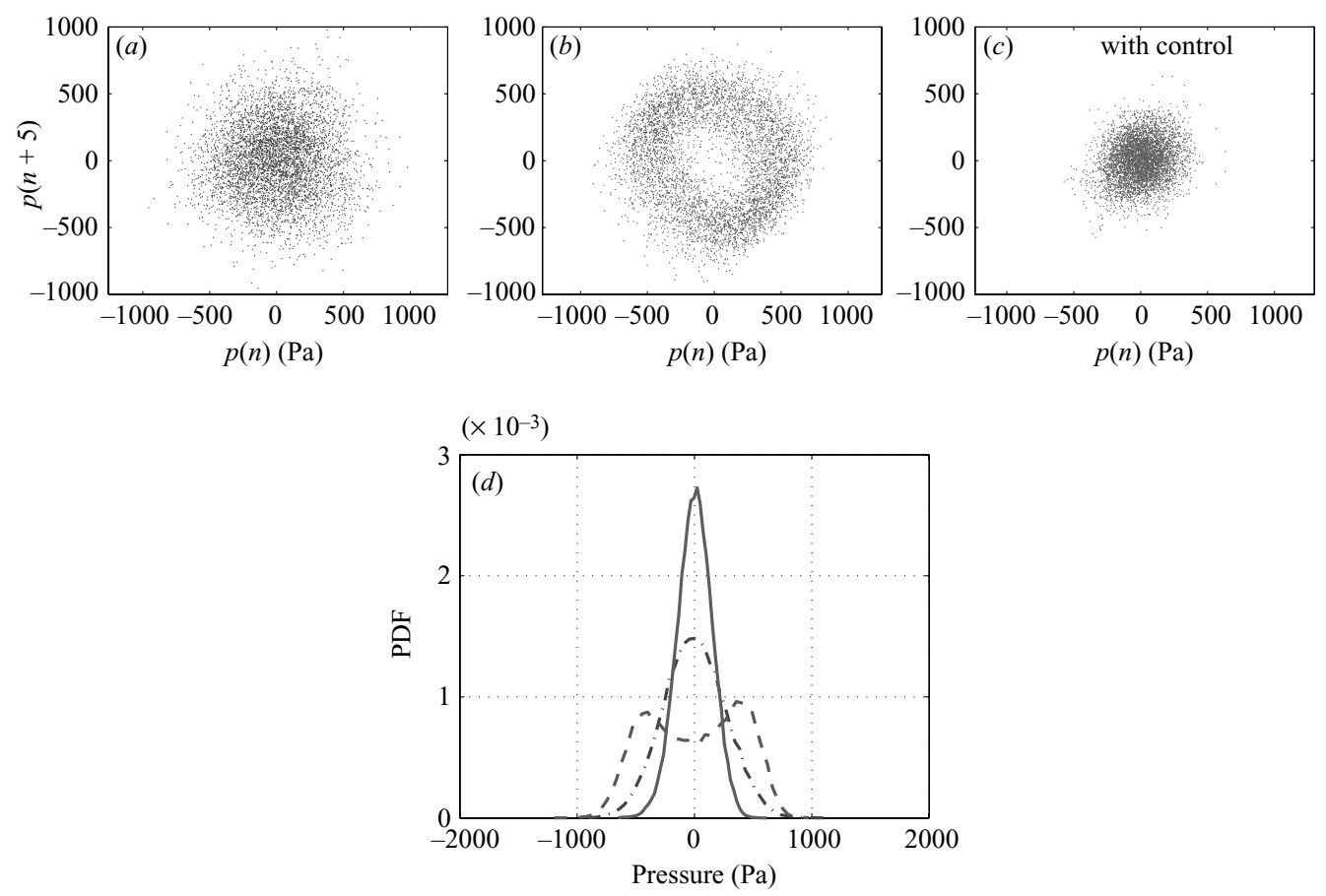

FIGURE 5. Phase portraits of pressure signal from Kulite 7 at Mach numbers $(a) M=0.45$, (b) $M=0.34$; and (c) at $M=0.34$ with feedback controller tuned to suppress oscillations. (d) PDFs for the three datasets: $M=0.45(-\cdot-), M=0.34$ unforced (-- -), and $M=0.34$ with control $(-)$ (gain $=4.0$ ). The $M=0.34$ case without control is in a limit cycle, but for the other cases, amplification of external disturbances is the dominant oscillation mechanism.

have more than one peak. For a more rigorous treatment of these ideas, and their application to a combustion control experiment, see Mezic \& Banaszuk (2004).

We can also use time-delay phase portraits to distinguish between the two regimes. The phase portrait of a limit cycling system will be a closed curve (or, with noise, a 'fuzzy' closed curve), while the phase portrait of a stable system forced by noise will be concentrated about a point. Thus, the phase portrait and the PDF are useful tools for distinguishing whether nonlinearities or external disturbances are more important: if two (or more) peaks are observed in the PDF of the bandpass filtered signal, then a limit cycle is present, and nonlinearities are dominant; if a single peak is observed, we cannot conclude whether a limit cycle is present (as it may be buried in the noise), but nevertheless the noise is the dominant feature.

Phase portraits for the same measurements as in figure 4 are shown in figure 5 . At $M=0.34$, the system appears to be in a limit cycle. The phase portrait indeed looks like a fuzzy ellipse, and the PDF has two distinct peaks. However, at $M=0.45$, the system appears to be at a stable equilibrium point, driven by noise. The phase portrait is concentrated about a point, and the PDF has a single peak which closely resembles a Gaussian.

A sweep of Mach numbers from 0.1 to 0.45 in increments varying from 0.01 to 0.05 revealed that $M=0.34$ is the only Mach number where the unstable regime is observed. Furthermore, at this Mach number, only a single frequency is observed, while at most other Mach numbers, multiple modes exist simultaneously. This is possibly because at $M=0.34$, the longitudinal acoustic modes in the cavity reinforce 
the Rossiter modes: the frequency of the first longitudinal mode coincides with the frequency of the third Rossiter mode (Williams et al. 2000). Presumably, this reinforcement increases the loop gain at this frequency enough to cause the system $(P$ in figure 1) to become unstable. Another possible mechanism put forth by Alvarez \& Kerschen (2005) suggests that the wind-tunnel walls increase the growth rate of specific acoustic modes.

We note that more generally, any effect which increases this loop gain may cause instability. In particular, Rowley et al. (2002) observed that cavities with laminar upstream boundary layers will experience greater shear-layer amplification, and thus a greater loop gain. This is further corroborated by Sarohia (1975), who observed that cavities with laminar upstream boundary layers were more likely to exhibit oscillations. This may explain why the simulations in Rowley et al. (2002) with laminar upstream boundary layers are apparently in the unstable regime, where the parameter values are otherwise similar to those in the present experiment.

\subsection{Behaviour with and without active suppression}

The oscillations were suppressed by feeding back the pressure signal from Kulite 8 through an analogue controller consisting of a bandpass filter and a phase shifter, used in previous experiments by Williams et al. (2000). Pressure measurements for the baseline and controlled cases are compared in figures 4 and 5. Here, the controller applies a gain of 4.0 , with a passband of $315-400 \mathrm{~Hz}$, and a phase shift of $89^{\circ}$ at the resonant frequency of $337 \mathrm{~Hz}$ (the gain and phase are measured between the pressure sensor feedback signal and the input to the actuator amplifier). From the phase portrait and the PDF, it appears that the unforced case is in a limit cycle, but with control there is a stable equilibrium point. The frequency response shows that the closed-loop system does excite oscillations at a new frequency (at $435 \mathrm{~Hz}$ ), and we discuss these adverse effects of control later, but from the PDF it appears that these oscillations are the result of disturbance amplification, not instability.

\section{Performance limits}

In this section, we use the analytical model developed in $\S 2$ to understand any fundamental limitations of feedback control, for the given arrangement of sensors and actuators.

\subsection{Empirically tuned model}

The model we use is given by equation (2.1), with parameters chosen to make the model agree approximately with the experimental conditions at $M=0.34$, and the resulting frequency response is shown in figure 6. Actual values of time delays were used where known, and the remaining constants were adjusted so that at least one pole would be unstable, as observed in the experiment. For the shear layer, equation (2.2) is used, with $\omega_{0}=350 \mathrm{~Hz}, \zeta=0.2$, and $\tau_{s}=L / c_{p}$, with $\kappa=c_{p} / U=0.625$. (Here, $U \approx 117.5 \mathrm{~m} \mathrm{~s}^{-1}$ is the free-stream velocity.) For the shear-layer time delay, we use a tenth-order Padé approximation to obtain a rational transfer function (for this time delay, the approximation is valid up to about $500 \mathrm{~Hz}$ ). The acoustics are modelled by equation (2.3) with $r=0.45$ and $\tau_{a}=L / a$, where $a$ is the sound speed in the free stream, and a fourth-order Padé approximation is used for the acoustic time delay (valid up to $800 \mathrm{~Hz}$ ). Variations in the sound speed are assumed small for this relatively low Mach number. The scattering gain is taken to be 0.23 , and changing this parameter adjusts the stability of the system: for larger values of this gain, the system is unstable, and for smaller values, the system is more heavily damped. 

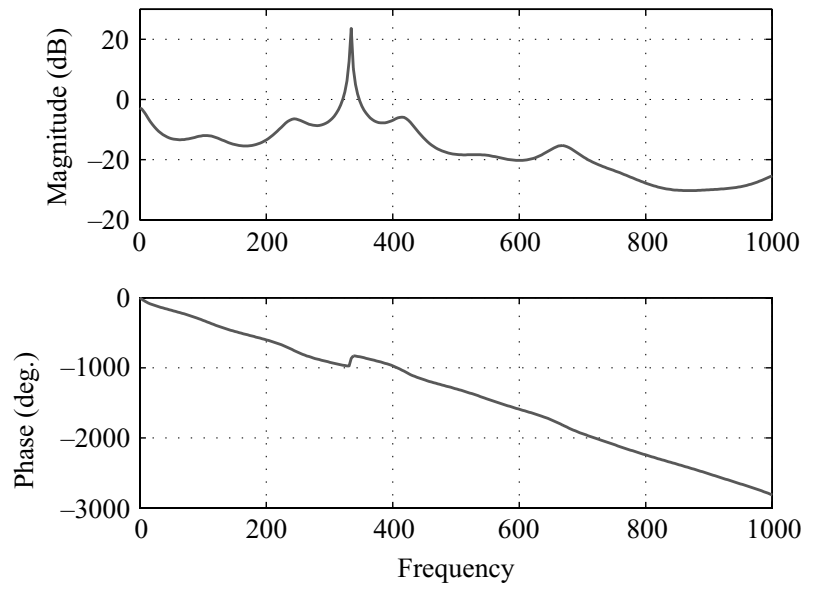

FIgURE 6. Magnitude and phase of $P(\mathrm{i} \omega)$ for cavity model at $M=0.34$.

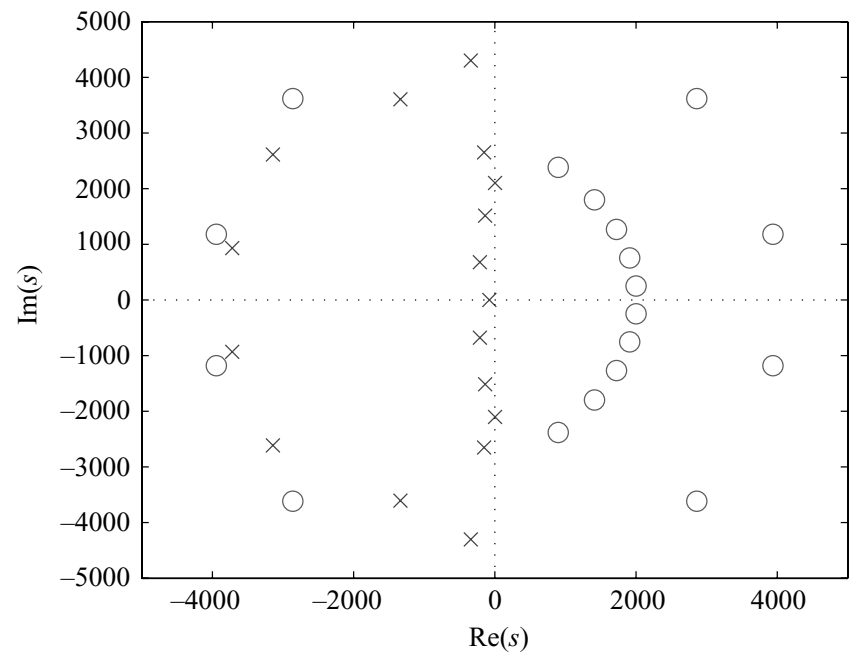

Figure 7. Poles $(\times)$ and zeros $(\bigcirc)$ of $P(s)$ at $M=0.34$, with Padé approximations for the time delays.

For a stable linear system, the magnitude of the frequency response may be viewed precisely as the amount the flow amplifies disturbances at each frequency. The model shown in figures 6 and 7 is unstable (the pole at $\operatorname{Im}(s) \approx 2100 \approx 334 \times 2 \pi$ is in the right half-plane), and so this interpretation does not literally hold for figure 6 , but is still helpful for understanding the closed-loop results, once a stabilizing controller is introduced, as will be discussed below. The peaks predicted by the model (imaginary parts of the poles near the imaginary axis) are at $108 \mathrm{~Hz}, 241 \mathrm{~Hz}, 334 \mathrm{~Hz}$ and $422 \mathrm{~Hz}$, which correspond to the first four Rossiter frequencies. (Note that imaginary parts of the poles correspond to frequencies in $\mathrm{rad} \mathrm{s}^{-1}$ ). In particular, the $334 \mathrm{~Hz}$ pole closely agrees with the $337 \mathrm{~Hz}$ peak measured experimentally; this pole is the most unstable in the model because the shear-layer amplification is the greatest for this frequency, and because the cavity acoustics reinforce oscillations at this frequency. For higher frequencies, the model ceases to be valid, because of the approximations for the time delays. 

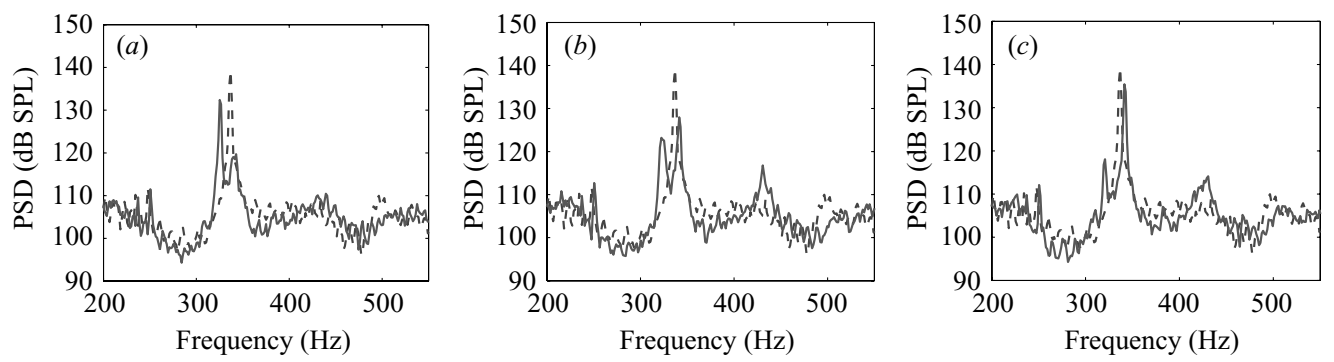

FiguRE 8. Power spectra of pressure signal from Kulite 8 at $M=0.34$, showing sidebands: control off $(--)$; control on $(-)($ gain $=7.8)$. (a) Delay $=4 \times 10^{-4} \mathrm{~s}$; (b) $5 \times 10^{-4} \mathrm{~s}$; (c) $6 \times$ $10^{-4} \mathrm{~s}$.

\subsection{Peak-splitting phenomenon}

In the spectra shown in figure 4 , at $M=0.34$ the controller is able to reduce the amplitude of the tone at $337 \mathrm{~Hz}$, but not eliminate it completely. Increasing the controller's gain does not result in further suppression, as shown in figure 8, which shows spectra from three different controllers with a larger gain $($ gain $=7.8)$ than that shown in figure 4 . These controllers were implemented digitally, but had the same structure as the analogue controller used in figure 4: a second-order Butterworth filter with a passband of $290-390 \mathrm{~Hz}$ was used, along with a time delay, which was varied as shown in figure 8 . The phase shifts (at $337 \mathrm{~Hz}$ ) of the bandpass filter with time delays of $0.4,0.5$ and $0.6 \mathrm{~ms}$ were $124^{\circ}, 97^{\circ}$ and $80^{\circ}$, respectively.

In figure 8 , the main resonant frequency at $337 \mathrm{~Hz}$ is almost completely attenuated, but sidebands appear very close in frequency, at about $320 \mathrm{~Hz}$ and $341 \mathrm{~Hz}$. As the time delay is changed, the relative strength of the sidebands changes, and the frequency changes slightly-the lower frequency shifts from 320 to $325 \mathrm{~Hz}$ in figures 8 (a)-8(c). In retrospect, this peak-splitting phenomenon also appears in some previous closedloop cavity experiments (e.g. Cattafesta et al. 1999, figure 5). This phenomenon has also been observed in combustion experiments at UTRC by Banaszuk et al. (1999) and Cohen \& Banaszuk (2003), where they have been explained using the linear mechanism discussed below.

To explain these effects, consider how feedback affects the amplification of disturbances. Without control, the transfer function from disturbances to measured pressure is simply $P(s)$ (see figure 1). With feedback, and assuming $V(s)=1$, the transfer function is $P(s) /(1+P(s) C(s))$, so the open-loop transfer function is modified by the amount

$$
S(s)=\frac{1}{1+P(s) C(s)},
$$

called the sensitivity function. If $|S(\mathrm{i} \omega)|<1$, then disturbances are attenuated, so feedback is beneficial, but if $|S(\mathrm{i} \omega)|>1$, then disturbances are amplified by control. Here, we will assume that the closed-loop system is stable, even though the open-loop $P(s)$ may be unstable (as it should be at $M=0.34$, from figure 5). Note that if $P$ is unstable, the open-loop amplification of disturbances is unbounded, but the closedloop amplification is still $|P(\mathrm{i} \omega) S(\mathrm{i} \omega)|$, so the Bode magnitude plot in figure 6 still has a meaningful interpretation. Also note that if there are actuator dynamics $(V(s) \neq 1)$, these may be included by incorporating $V(s)$ into $P(s)$.

The sensitivity function may be determined from a Nyquist plot of the system, which is just a plot of $P(\mathrm{i} \omega) C(\mathrm{i} \omega)$ in the complex plane, as $\omega$ varies from $0 \rightarrow \infty$. 


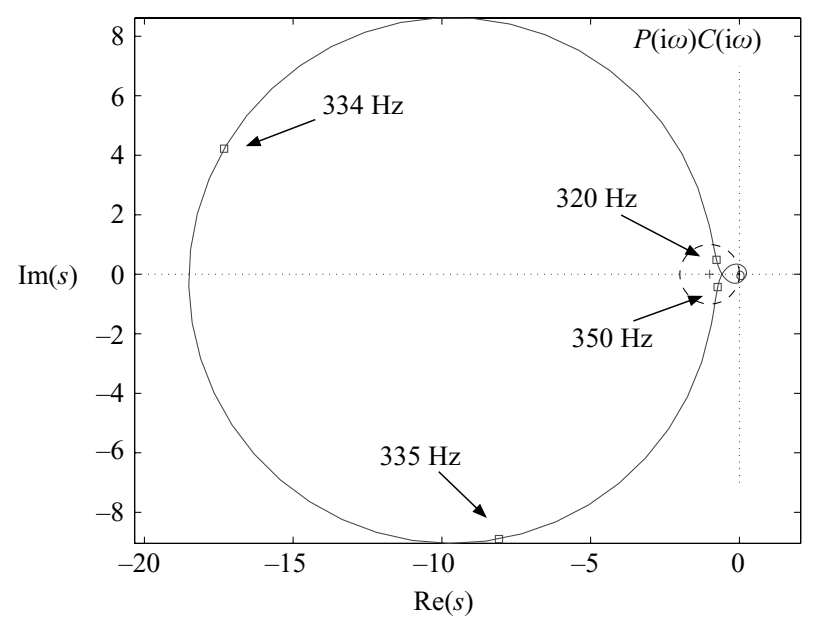

FIGURE 9. Nyquist plot for the feedback system, along with corresponding frequencies (in $\mathrm{Hz}$ ). Points outside the dashed circle correspond to a performance benefit $(|S(\mathrm{i} \omega)|<1)$, and points inside the circle correspond to a penalty $(|S(\mathrm{i} \omega)|>1)$.

Figure 9 shows the Nyquist plot for the plant $P(s)$ given by the empirically tuned model shown in figure 6 , for $C(s)$ given by a bandpass filter, with a gain and time delay. Graphically, the magnitude of the sensitivity function $S(\mathrm{i} \omega)$ is the reciprocal of the distance from $P(\mathrm{i} \omega) C(\mathrm{i} \omega)$ to the -1 point. Thus, from figure 9 we would expect the peak frequency of $334 \mathrm{~Hz}$ to be attenuated, since this point is far from the -1 point, while frequencies at $320 \mathrm{~Hz}$ and $350 \mathrm{~Hz}$ should be amplified by the feedback loop, as these points on the Nyquist plot lie inside the unit circle centred about the -1 point, and thus correspond to $|S(\mathrm{i} \omega)|>1$. As expected, feedback attenuates the main frequency, but amplifies sidebands.

If the gain is increased, the entire Nyquist plot is magnified, so the $334 \mathrm{~Hz}$ peak moves farther from the -1 point, while the sidebands move closer, so the main frequency should be attenuated more, while the side peaks should be amplified more. This effect is also consistent with the observed experimental behaviour.

For a slightly smaller time delay than that used in figure 9 the Nyquist plot will be rotated slightly counterclockwise, so the lower-frequency sideband at $320 \mathrm{~Hz}$ will be amplified more, while the higher-frequency sideband at $350 \mathrm{~Hz}$ will not be amplified as much. Conversely, for a slightly larger time delay, the Nyquist plot will be rotated clockwise, so the $350 \mathrm{~Hz}$ sideband should be amplified more. These effects are all consistent with the experimental results shown in figure 8 .

Note also that a controller-induced resonance at $435 \mathrm{~Hz}$ is present for the analogue controller shown in figure 4, but much less significant for the digital controller in figure 8 . This is because the passbands of the analogue and digital controllers are different, and the digital controller rolls off more steeply, so the gain at $435 \mathrm{~Hz}$ is smaller than for the analogue controller.

\subsection{Fundamental limitations of closed-loop control}

Ideally, we would like to design a compensator $C(s)$ such that the sensitivity function $|S(\mathrm{i} \omega)| \ll 1$ for all frequencies. Unfortunately, this is not possible, because of Bode's integral formula (also known as the area rule), which states that under weak assumptions, any decrease in sensitivity over one frequency range must be balanced by an increase for some other frequencies (Doyle, Francis \& Tannenbaum 1992). 
More precisely, for a system with relative degree at least 2, the area rule states that

$$
\int_{0}^{\infty} \log |S(\mathrm{i} \omega)| \mathrm{d} \omega=\pi \sum_{k} \operatorname{Re}\left(p_{k}\right),
$$

where $p_{k}$ are the unstable poles of $P C$. So for a stable plant, any negative area $(\mid S \mathrm{i} \omega) \mid<1)$ in the $\log$-linear plot of $S$ versus $\omega$ must be balanced by an equal positive area $(|S(\mathrm{i} \omega)|>1)$, no matter how the controller $C(s)$ is chosen. For unstable plants, the situation is worse, and the net area must be positive.

The area rule in itself does not imply any peaking of $|S(\mathrm{i} \omega)|$, as the positive area may be spread out over a large frequency band, as $\omega \rightarrow \infty$. However, Banaszuk et al. (1999) and Cohen \& Banaszuk (2003) showed that for narrow bandwidth controllers, and plants with significant time delays, the area rule does imply a peaking of $|S(\mathrm{i} \omega)|$, since all of the amplification must occur within the narrow bandwidth of the controller. The narrower the bandwidth, or the longer the time delay, the greater the amount of peaking. This implies a strong argument in favour of large bandwidth actuators, and suggests that narrow-bandwidth actuators (such as piezoelectrics) might not be suitable for feedback control.

\section{Conclusions}

We have presented a linear model for cavity oscillations, incorporating the effect of external disturbances. Under some conditions, the system is unstable, and perturbations will grow until nonlinearities become important and the linear model is no longer valid. However, for other conditions, the system is stable, but lightly damped, acting as a noise amplifier. Phase portraits and probability density functions of experimental data indicate that for most flow regimes observed in our experiment, the cavity is a stable noise amplifier, oscillating at several different Rossiter modes simultaneously. For the $M=0.34$ case, however, the flow is in a limit cycle, oscillating at a single Rossiter mode, possibly because of enhanced acoustic resonance at this value of $M$.

For this Mach number, the flow may be stabilized using a controller consisting of a bandpass filter and time delay. When control is introduced, however, a peak-splitting phenomenon is observed, in which the main peak splits into two sidebands. These same effects are found in the linear model. The peak splitting effect has been observed in experiments in combustion instabilities by Banaszuk et al. (1999), and is a common feature of systems with limited bandwidth and large time delay.

If the noise-amplification model of cavity oscillations is correct, we cannot expect to be able to reduce the amplitude of oscillations at all frequencies using feedback, because of fundamental limitations imposed by the area rule. However, given an accurate model of the system (e.g. from a frequency response experiment), it is straightforward to design a compensator to minimize these adverse effects, and reduce oscillations over important frequency ranges, while paying the penalty over less important frequency ranges, or ranges where the plant itself is not so sensitive to disturbances.

Finally, we note that while nonlinearities may be important for cavity oscillations in other parameter ranges, it is hoped the controlled system will always be stable, so the linear models presented here should always be useful for understanding the controlled system. 
This work was originally presented as an AIAA conference paper (AIAA 2002-0972) at the 2002 Aerospace Sciences meeting. We would like to thank Andrzej Banaszuk for many helpful comments, and for suggesting the method for distinguishing between stable and unstable noisy systems. The theoretical work was supported by AFOSR under grants F49620-98-1-0095 and F49620-03-1-0081 with program managers Dr Thomas Beutner and Dr Sharon Heise. The experimental work was supported by AFOSR under grants F49620-98-1-0276 and F49620-03-1-0074, with program managers Dr Steve Walker and Dr John Schmisseur.

\section{REFERENCES}

Alvarez, J. O. \& Kerschen, E. J. 2005 Influence of wind tunnel walls on cavity acoustic resonances. 11th AIAA/CEAS Aeroacoustics Conf. AIAA Paper 2005-2804.

Banaszuk, A., Jacobson, C. A., Khibnik, A. I. \& Mehta, P. G. 1999 Linear and nonlinear analysis of controlled combustion processes. Part I: Linear analysis. Proc. of Conf. on Control Applications, Kamuela, HI, USA.

Cattafesta, III, L. N., Shukla, D., Garg, S. \& Ross, J. A. 1999 Development of an adaptive weapons-bay suppression system. AIAA Paper 99-1901.

Cohen, J. M. \& Banaszuk, A. 2003 Factors affecting the control of unstable combustors. J. Prop. Power 19, 811-821.

Crighton, D. G. 1992 The jet edge-tone feedback cycle; linear theory for the operating stages. J. Fluid Mech. 234, 361-391.

Doyle, J. C., Francis, B. A. \& Tannenbaum, A. R. 1992 Feedback Control Theory. Macmillan.

Huerre, P. \& Monkewitz, P. A. 1990 Local and global instabilities in spatially developing flows. Annu. Rev. Fluid Mech. 22, 473-537.

Kerschen, E. J. \& Tumin, A. 2003 A theoretical model of cavity acoustic resonances based on edge scattering processes. AIAA Paper 2003-0175.

Mezic, I. \& Banaszuk, A. 2004 Comparison of systems with complex behaviour. Physica D 197, $101-133$.

Rossiter, J. E. 1964 Wind-tunnel experiments on the flow over rectangular cavities at subsonic and transonic speeds. Aeronaut. Res. Council Rep. Mem. 3438.

Rowley, C. W., Colonius, T. \& Basu, A. J. 2002 On self-sustained oscillations in two-dimensional compressible flow over rectangular cavities. J. Fluid Mech. 455, 315-346.

SARoHiA, V. 1975 Experimental and analytical investigation of oscillations in flows over cavities. $\mathrm{PhD}$ thesis, California Institute of Technology.

Smith, B. L. \& Glezer, A. 1998 The formation and evolution of synthetic jets. Phys. Fluids 10, 2281-2297.

TAM, C. K. W. \& Block, P. J. W. 1978 On the tones and pressure oscillations induced by flow over rectangular cavities. J. Fluid Mech. 89, 373-399.

Williams, D. R., Fabris, D. \& Morrow, J. 2000 Experiments on controlling multiple acoustic modes in cavities. AIAA Paper 2000-1903. 